\title{
COUPLED AND TRIPLED FIXED POINT THEOREMS ON A METRIC SPACE ENDOWED WITH A BINARY RELATION
}

\author{
MELÁNIA-IULIA DOBRICAN \\ Received 28 November, 2015
}

\begin{abstract}
The purpose of this paper is to transpose some of the results regarding the coupled fixed point theory, developed by Bhaskar and Lakshmikantham [7], using the approaches of Ben-El-Mechaiekh [2], Asgari, Mousavi [1] and Berinde [3]. The first approach replaces the partial order relation from the Ran-Reurings fixed point theorem [10] with a transitive one, the second one uses a reflexive-only relation and whereas the third one introduces the tripled fixed points. Our aim is to obtain some generalized fixed point theorems, containing a more simple symmetrical condition, also extending them for the case of tripled fixed point.
\end{abstract}

2010 Mathematics Subject Classification: $47 \mathrm{H} 10$

Keywords: coupled fixed point, tripled fixed point, symmetrical contractivity condition, binary relation, reflexivity

\section{INTRODUCTION}

Ran and Reurings [10], in 2003, extended the famous fixed point theorem of Banach, endowing the metric space $X$ with a partial order relation. Thus, the contractivity condition wasn't satisfied unless the elements $x$ and $y$ chosen from the metric space were comparable (i.e. $x \geq y$ ). Their work inspired many others who started studying the existence and uniqueness of fixed points in partially ordered metric spaces.

In 2006 Bhaskar and Lakshmikantham [7] have studied the coupled fixed points introduced by Guo and Lakshmikantham [8], in the context of a weak contractive condition, providing some fixed point theorems with respect to it.

These results, at their turn, produced enthusiasm among the mathematicians who continued studying the simple and coupled fixed point, obtaining new important results, with applications in different types of equations. In 2011 Berinde [3] extends the coupled fixed point theorems for mixed monotone operators obtained by Bhaskar and Lakshmikantham by significantly weakening the contractivity condition involved. $\mathrm{He}$ also introduces a new concept: the tripled fixed point, in collaboration with Borcut [4]. 
In 2012, Samet and Turinici [11] establish fixed point theorems for contractive mappings on a metric space endowed with an amorphous binary relation. They also show that some fixed point theorems for cyclical contractive mappings can be deduced from their main results.

Ben-el-Mechaiekh [2] observes that, in the proof of Ran and Reurings fixed point theorem, neither the antisymmetry, nor the reflexivity of the partial order relation are being used. In 2014 he replaces the relation used by the two mathematicians with a transitive binary relation. His work is essentially based on the extension that Edelstein brings to Banach's contraction principle in 1961 [6].

One of the latest results in this field is provided by Asgari and Mousavi [1] who replaced the partial order relation used by Ran and Reurings, with a reflexive relation that is not necessarily neither transitive, nor antisymmetric. Their paper was published in the spring of 2015 and the key feature of their results is the fact that the contractivity condition holds only on comparable elements with respect to the binary relation.

\section{RESULtS}

\subsection{Preliminary considerations}

Now, we start by recalling the definition of (R-)coupled and tripled fixed point; see for instance [7], [4], [1].

Definition 1 ([7]). We call an element $(x, y) \in X \times X$ a coupled fixed point of the mapping $F$, if $F(x, y)=x$ and $F(y, x)=y$.

Definition 2 ([1]). Let $X$ be a nonempty set and let $R$ be a reflexive relation on $X, f: X \times X \rightarrow X$. An element $(x, y) \in X \times X$ is called $R$-coupled fixed point of $f$, if $f \times f(x, y) \in X_{R}(x, y)$.

Definition 3 ([4]). We call an element $(x, y, z) \in X \times X$ a tripled fixed point of the mapping $F$, if $F(x, y, z)=x, F(y, x, z)=y$ and $F(z, y, x)=z$.

Asgari and Mousavi [1] also redefine the mixed monotone property of a mapping, introduced by Bhaskar and Lakshmikantham [7], endowing the metric space with a reflexive relation. This is given as follows:

Definition 4 ([1]). Let $X$ be a nonempty set and let $R$ be a reflexive relation on $X, f: X \times X \rightarrow X$. The mapping $f$ has the mixed $R$-monotone property on $X$ if $f \times f\left(X_{R}(x, y)\right) \subseteq X_{R}(f \times f(x, y))$, for all $(x, y) \in X \times X$, where $X_{R}(x, y)=$ $\{(z, t) \in X \times X: z R x \wedge y R t\}, \forall(x, y) \in X \times X$.

In [1] Asgari and Mousavi also prove a coupled fixed point theorem with respect to a reflexive relation.

Theorem 1 ([1]). Let $(X, d)$ be a metric space and $R$ a reflexive relation on $X$. If $f: X \times X \rightarrow X$ is a mapping such that: 
- $f$ has the mixed $R$-monotone property on $X$.

- $(X, d)$ is a complete metric space.

- $f$ has an $R$-coupled fixed point, i.e. there exists $\left(x_{0}, y_{0}\right) \in X \times X$ such that $f \times f\left(x_{0}, y_{0}\right) \in X_{R}\left(x_{0}, y_{0}\right)$.

- there exists a constant $k \in[0,1)$ such that:

$$
d(f(x, y), f(z, t)) \leq \frac{k}{2}[d(x, z)+d(y, t)], \forall(x, y) \in X_{R}(z, t) .
$$

- $f$ is orbitally continuous.

Then:

- There exists $x^{*}, y^{*} \in X$ such that $f\left(x^{*}, y^{*}\right)=x^{*}$ and $f\left(y^{*}, x^{*}\right)=y^{*}$.

- The sequences $\left\{x_{n}\right\}_{n \in \mathbb{N}}$ and $\left\{y_{n}\right\}_{n \in \mathbb{N}}$ defined by $x_{n+1}=f\left(x_{n}, y_{n}\right)$ and $y_{n+1}=f\left(y_{n}, x_{n}\right)$ converge respectively to $x^{*}$ and $y^{*}$.

- The error estimation is given by:

$$
\begin{gathered}
\max _{n \in \mathbb{N}}\left\{d\left(x_{n}, x^{*}\right), d\left(y_{n}, y^{*}\right)\right\} \leq \\
\frac{k^{n}}{2(1-k)}\left[d\left(f\left(x_{0}, y_{0}\right), x_{0}\right)+d\left(f\left(y_{0}, x_{0}\right), y_{0}\right)\right] .
\end{gathered}
$$

In [9], Rus and Petrușel emphasize the fact that, when working on a set that is endowed with a metric and a binary relation (an order structure in [9]), we should add an additional assumption, namely the compatibility between the two structures:

Remark 1 ([9]). If $\left\{x_{n}\right\}_{n \in \mathbb{N}} \rightarrow x,\left\{y_{n}\right\}_{n \in \mathbb{N}} \rightarrow y$ and $x_{n} \leq y_{n}$ then $x \leq y, \forall n \in \mathbb{N}$.

Thus, the compatibility condition between the metric and the reflexive relation $R$ used in the theorem above [1] should be :

Remark 2. If $x_{n} R y_{n}$ then $\lim _{n \rightarrow \infty} x_{n} R \lim _{n \rightarrow \infty} y_{n}, \forall n \in \mathbb{N}$.

\subsection{A coupled fixed point theorem}

The following result generalizes the main result of Asgari and Mousavi [1], using a more general symmetrical condition, like the one used by Berinde [3].

Theorem 2. Let $(X, d)$ be a metric space, $R$ be a binary reflexive relation on $X$ such that $R$ and $d$ are compatible. If $F: X^{2} \rightarrow X$ is a mapping having an $R$ coupled fixed point, the mixed $R$-monotone property on $X$, it is orbitally continuous and $\exists k \in[0,1)$ such that

$$
\begin{gathered}
d(F(x, y), F(z, t))+d(F(y, x), F(t, z)) \leq k[d(x, z)+d(y, t)], \\
\forall(x, y) \in X_{R}(z, t) .
\end{gathered}
$$

Then:

(1) $\exists(\bar{x}, \bar{y}) \in X^{2}$ such that $F(\bar{x}, \bar{y})=\bar{x}$ and $F(\bar{y}, \bar{x})=\bar{y}$.

(2) The sequences $\left\{x_{n}\right\}_{n \in \mathbb{N}},\left\{y_{n}\right\}_{n \in \mathbb{N}}$, defined by $x_{n+1}=F\left(x_{n}, y_{n}\right), y_{n+1}=$ $F\left(y_{n}, x_{n}\right)$, converge to $\bar{x}$ and $\bar{y}$, respectively. 
(3) The error estimation that holds is:

$$
\max _{n \in \mathbb{N}}\left\{d\left(x_{n}, \bar{x}\right), d\left(y_{n}, \bar{y}\right)\right\} \leq \frac{k^{n}}{1-k}\left[d\left(f\left(x_{0}, y_{0}\right), x_{0}\right)+d\left(f\left(y_{0}, x_{0}\right), y_{0}\right)\right] .
$$

Proof. Since the mapping $F$ admits an $R$-coupled fixed point, let $\left(x_{0}, y_{0}\right) \in X \times X$ be it, we have $F \times F\left(x_{0}, y_{0}\right) \in X_{R}\left(x_{0}, y_{0}\right)$. Further, using the mixed $R$-monotone property of $F$, we have $F \times F\left(x_{0}, y_{0}\right) \in X_{R}\left(F\left(x_{0}, y_{0}\right), F\left(y_{0}, x_{0}\right)\right)$. Using the induction, we can easily prove that:

$$
\left(F^{n}\left(x_{0}, y_{0}\right), F^{n}\left(y_{0}, x_{0}\right)\right) \in X_{R}\left(F^{n-1}\left(x_{0}, y_{0}\right), F^{n-1}\left(y_{0}, x_{0}\right)\right) .
$$

We define $d_{2}: X^{2} \times X^{2} \rightarrow \mathbb{R}_{+} d_{2}(Y, Z)=\frac{1}{2}[d(x, z)+d(y, t)], \forall Y=(x, y), Z=$ $(z, t) \in X^{2}$.

$d_{2}$ is a metric on $X^{2}$ because:

- $d_{2}(Y, Z)=0 \Leftrightarrow Y=Z$ is a simple task to check, using the definition of $d_{2}$ and the fact that $d$ is a metric.

- $d_{2}(Y, Z)=d_{2}(Z, Y), \forall Y, Z \in X^{2}$ holds, because $d$ is a metric, and the sum in $d_{2}$ 's definition is commutative.

- $d_{2}(Y, Z) \leq d_{2}(Y, T)+d_{2}(T, Z), \forall Y, T, Z \in X^{2}$ can also be easily checked.

Therefore $\left(X^{2}, d_{2}\right)$ is a complete metric space.

We consider the operator:

$T: X^{2} \rightarrow X^{2}$ defined by $T(Y)=(F(x, y), F(y, x)), \forall Y=(x, y) \in X^{2}$.

For $Y=(x, y), Z=(z, t) \in X^{2}$, considering the definition for $d_{2}$, we have:

$$
d_{2}(T(Y), T(Z))=\frac{d(F(x, y), F(z, t))+d(F(y, x), F(t, z))}{2}
$$

and

$$
d_{2}(Y, Z)=\frac{d(x, z)+d(y, t)}{2} .
$$

By the contractivity condition (2.1) we have

$$
d_{2}(T(Y), T(Z)) \leq k \cdot d_{2}(Y, Z), \forall Y, Z \in X^{2}, Y \geq Z .
$$

From (2.2), we have the monotony of $T$ and that $\left\{Z_{n}\right\}_{n \geq 0}-$ is nondecreasing, we denote $Z_{n}=\left(F^{n-1}\left(x_{0}, y_{0}\right), F^{n-1}\left(y_{0}, x_{0}\right)\right)$.

We denote $Y=Z_{n} \geq Z_{n-1}=V$.

We replace this in $(2.3)$, obtaining:

$$
\begin{gathered}
d_{2}\left(T\left(Z_{n}\right), T\left(Z_{n-1}\right)\right) \leq k \cdot d_{2}\left(Z_{n}, Z_{n-1}\right), n \geq 1 \Leftrightarrow \\
\Leftrightarrow d_{2}\left(Z_{n+1}, Z_{n}\right) \leq k \cdot d_{2}\left(Z_{n}, Z_{n-1}\right), n \geq 1 .
\end{gathered}
$$

Using the induction, we have:

$$
d_{2}\left(Z_{n+1}, Z_{n}\right) \leq k^{n} \cdot d_{2}\left(Z_{1}, Z_{0}\right), n \geq 1
$$


Let $i<j$. We get:

$$
\begin{gathered}
d_{2}\left(Z_{i}, Z_{j}\right) \leq \sum_{l=i+1}^{j} d_{2}\left(Z_{l}, Z_{l-1}\right) \leq\left(k^{i}+k^{i+1}+\ldots+k^{j-i-1}\right) \cdot d_{2}\left(Z_{1}, Z_{0}\right) \leq \\
\leq k^{i} \frac{1-k^{j-i-1}}{1-k} \cdot d_{2}\left(Z_{1}, Z_{0}\right)
\end{gathered}
$$

$\Rightarrow\left\{Z_{n}\right\}_{n \geq 0}$ is a Cauchy sequence in the complete metric space $\left(X^{2}, d_{2}\right) \Rightarrow$

$$
\Rightarrow \lim _{n \rightarrow \infty} Z^{n}=\bar{Z} \text {. }
$$

We now use (2.2): $T(\bar{Z})=\bar{Z} \Leftrightarrow(F(\bar{x}, \bar{y}), F(\bar{y}, \bar{x}))=(\bar{x}, \bar{y})$ $\Leftrightarrow F(\bar{x}, \bar{y})=\bar{x}, F(\bar{y}, \bar{x})=\bar{y} \Leftrightarrow(\bar{x}, \bar{y})$ is the coupled fixed point for $F$.

Since $(X, d)$ is a complete metric space, $\exists \bar{x}, \bar{y} \in X$ such that $F^{n}\left(x_{0}, y_{0}\right) \rightarrow \bar{x}, F^{n}\left(y_{0}, x_{0}\right) \rightarrow \bar{y}, \quad n \rightarrow \infty$. Using the fact that $F$ is orbitally continuous, we have:

$$
\begin{aligned}
& \left\{x_{n}\right\}_{n \in \mathbb{N}} \rightarrow \bar{x}, x_{n+1}=F\left(x_{n}, y_{n}\right) \\
& \left\{y_{n}\right\}_{n \in \mathbb{N}} \rightarrow \bar{y}, y_{n+1}=F\left(y_{n}, x_{n}\right)
\end{aligned}
$$

So, by (2.4) we have:

$$
d_{2}\left(\left(x_{n}, y_{n}\right),(\bar{x}, \bar{y})\right) \leq \frac{k^{n}}{1-k} \cdot d_{2}\left(\left(x_{1}, y_{1}\right),\left(x_{0}, y_{0}\right)\right), n \geq 0 .
$$

We return to the original metric $d$ :

$$
\begin{gathered}
\frac{d\left(x_{n}, \bar{x}\right)+d\left(y_{n}, \bar{y}\right)}{2} \leq \frac{k^{n}}{1-k} \cdot \frac{d\left(x_{1}, x_{0}\right)+d\left(y_{1}, y_{0}\right)}{2} \Leftrightarrow \\
\Leftrightarrow d\left(x_{n}, \bar{x}\right)+d\left(y_{n}, \bar{y}\right) \leq \max _{n \in \mathbb{N}}\left\{d\left(x_{n}, \bar{x}\right), d\left(y_{n}, \bar{y}\right)\right\} \leq \frac{k^{n}}{1-k} \cdot\left[d\left(x_{1}, x_{0}\right)+d\left(y_{1}, y_{0}\right)\right] . \\
\text { But } x_{n+1}=F\left(x_{n}, y_{n}\right) \text { and } y_{n+1}=F\left(y_{n}, x_{n}\right) . \text { We get: } \\
\max _{n \in \mathbb{N}}\left\{d\left(x_{n}, \bar{x}\right), d\left(y_{n}, \bar{y}\right)\right\} \leq \frac{k^{n}}{1-k} \cdot\left[d\left(F\left(x_{0}, y_{0}\right), x_{0}\right)+d\left(F\left(y_{0}, x_{0}\right), y_{0}\right)\right] .
\end{gathered}
$$

\subsection{A tripled fixed point theorem}

We now extend the results of Asgari and Mousavi [1], using the tripled fixed points concept introduced by Berinde and Borcut [4]:

Definition 5. Let $X$ be a topological space and $f, g: X \times X \times X \rightarrow X$. Then:

- An element $(x, y, z) \in X \times X \times X$ is called a tripled attractor basin element of $f$ with respect to $\left(x^{*}, y^{*}, z^{*}\right) \in X \times X \times X$, if $f^{n}(x, y, z) \rightarrow x^{*}$, $f^{n}(y, x, z) \rightarrow y^{*}$ and $f^{n}(z, x, y) \rightarrow z^{*}$, when $n \rightarrow \infty$. The set of these points $\left(x^{*}, y^{*}, z^{*}\right)$ will be denoted with $A_{f}^{c}\left(x^{*}, y^{*}, z^{*}\right)$, and for $x^{*}$, with $A_{f}\left(x^{*}\right)$. 
- The mapping $f$ is called orbitally continuous if $(x, y, z),(a, b, c) \in X \times$ $X \times X$ and $f^{n_{k}}(x, y, z) \rightarrow a, f^{n_{k}}(y, x, z) \rightarrow b, f^{n_{k}}(z, x, y) \rightarrow c$, when $k \rightarrow \infty$, implies $f^{n_{k}+1}(x, y, z) \rightarrow f(a, b, c), f^{n_{k}+1}(y, x, z) \rightarrow f(b, a, c)$ and $f^{n_{k}+1}(z, x, y) \rightarrow f(c, a, b)$, when $k \rightarrow \infty$.

- Let $X$ be a nonempty set and let $R$ be a reflexive relation on $X$. For every $(t, u, v) \in X \times X \times X$ we define $X_{R}(t, u, v)=\{(x, y, z) \in X \times X \times X: x R t \wedge$ $u R y \wedge z R v\}$.

Definition 6. Let $X$ be a nonempty set and let $R$ be a reflexive relation on $X$, $f: X \times X \times X \rightarrow X$.

- The mapping $f$ has the mixed $R$-monotone property on $X$ if $f \times f\left(X_{R}(x, y, z)\right) \subseteq X_{R}(f \times f(x, y, z))$, for all $(x, y, z) \in X \times X \times X$.

- An element $(x, y, z) \in X \times X \times X$ is called $R$-tripled fixed point of $f$, if $f \times f(x, y, z) \in X_{R}(x, y, z)$.

Theorem 3. Let $(X, d)$ be a metric space and $R$ be a binary reflexive relation on $X$ such that $R$ and $d$ are compatible. If $F: X^{3} \rightarrow X$ is a mapping having an $R$ tripled fixed point, the mixed $R$-monotone property on $X$, it is orbitally continuous and $\exists k \in[0,1)$ such that

$$
\begin{gathered}
d(F(x, y, z), F(t, u, v)) \leq k \cdot[d(x, t)+d(y, u)+d(z, v)] \\
\forall(x, y, z) \in X_{R}(t, u, v), k<1 .
\end{gathered}
$$

Then:

(1) $\exists(\bar{x}, \bar{y}, \bar{z}), \in X^{3}$ such that $F(\bar{x}, \bar{y}, \bar{z})=\bar{x}, F(\bar{y}, \bar{x}, \bar{z})=\bar{y}, F(\bar{z}, \bar{y}, \bar{x})=\bar{z}$.

(2) The sequences $\left\{x_{n}\right\}_{n \in \mathbb{N}},\left\{y_{n}\right\}_{n \in \mathbb{N}},\left\{z_{n}\right\}_{n \in \mathbb{N}}$, defined by $x_{n+1}=F\left(x_{n}, y_{n}, z_{n}\right), y_{n+1}=F\left(y_{n}, x_{n}, z_{n}\right), z_{n+1}=F\left(z_{n}, y_{n}, x_{n}\right)$, converge to $\bar{x}, \bar{y}$ and $\bar{z}$, respectively.

(3) The error estimation that holds is: $\max _{n \in \mathbb{N}}\left\{d\left(x_{n}, \bar{x}\right), d\left(y_{n}, \bar{y}\right), d\left(z_{n}, \bar{z}\right)\right\}$ $\leq \frac{k^{n}}{1-k}\left[d\left(F\left(x_{0}, y_{0}, z_{0}\right), x_{0}\right)+d\left(F\left(y_{0}, x_{0}, z_{0}\right), y_{0}\right)+d\left(F\left(z_{0}, y_{0}, x_{0}\right), z_{0}\right)\right]$.

Proof. Since the mapping $F$ admits an $R$-tripled fixed point, let $\left(x_{0}, y_{0}, z_{0}\right) \in$ $X \times X \times X$ be it, we have $F \times F\left(x_{0}, y_{0}, z_{0}\right) \in X_{R}\left(x_{0}, y_{0}, z_{0}\right)$. Further, using the mixed $R$-monotone property of $F$, we have

$$
F \times F\left(x_{0}, y_{0}, z_{0}\right) \in X_{R}\left(F\left(x_{0}, y_{0}, z_{0}\right), F\left(y_{0}, x_{0}, z_{0}\right), F\left(z_{0}, y_{0}, x_{0}\right)\right) .
$$

Using the induction, we can easily prove that:

$$
\begin{gathered}
\left(F^{n}\left(x_{0}, y_{0}, z_{0}\right), F^{n}\left(y_{0}, x_{0}, z_{0}\right), F^{n}\left(z_{0}, y_{0}, x_{0}\right)\right) \in \\
\in X_{R}\left(F^{n-1}\left(x_{0}, y_{0}, z_{0}\right), F^{n-1}\left(y_{0}, x_{0}, z_{0}\right), F^{n-1}\left(z_{0}, y_{0}, x_{0}\right)\right) .
\end{gathered}
$$

We claim that, for $n \in \mathbb{N}$

$$
\begin{array}{r}
d\left(F^{n+1}\left(x_{0}, y_{0}, z_{0}\right), F^{n}\left(x_{0}, y_{0}, z_{0}\right)\right) \leq \\
k^{n} \cdot\left[d\left(F\left(x_{0}, y_{0}, z_{0}\right), y_{0}\right)+d\left(F\left(y_{0}, z_{0}, x_{0}\right), y_{0}\right)+d\left(F\left(z_{0}, x_{0}, y_{0}\right), z_{0}\right)\right] .
\end{array}
$$


For $n=1$, we get:

$$
\begin{aligned}
d\left(F^{2}\left(x_{0}, y_{0}, z_{0}\right), F\left(x_{0}, y_{0}, z_{0}\right)\right)= & d\left(F\left(F\left(x_{0}, y_{0}, z_{0}\right), F\left(y_{0}, z_{0}, x_{0}\right), F\left(z_{0}, x_{0}, y_{0}\right)\right),\right. \\
& \left.F\left(x_{0}, y_{0}, z_{0}\right)\right) \\
\leq & k \cdot\left[d\left(F\left(x_{0}, y_{0}, z_{0}\right), x_{0}\right)+d\left(F\left(y_{0}, z_{0}, x_{0}\right), y_{0}\right)+d\left(F\left(z_{0}, x_{0}, y_{0}\right), z_{0}\right)\right] .
\end{aligned}
$$

Now, we assume that (2.7) holds. Using the forth assumption from the hypothesis we get:

$$
\begin{gathered}
d\left(F^{n+2}\left(x_{0}, y_{0}, z_{0}\right), F^{n+1}\left(x_{0}, y_{0}, z_{0}\right)\right)= \\
d\left(F\left(F^{n+1}\left(x_{0}, y_{0}, z_{0}\right), F^{n+1}\left(y_{0}, z_{0}, x_{0}\right), F^{n+1}\left(z_{0}, x_{0}, y_{0}\right)\right),\right. \\
\left.F\left(F^{n}\left(x_{0}, y_{0}, z_{0}\right), F^{n}\left(y_{0}, z_{0}, x_{0}\right), F^{n}\left(z_{0}, x_{0}, y_{0}\right)\right)\right) \leq \\
\leq k \cdot\left[d\left(F^{n+1}\left(x_{0}, y_{0}, z_{0}\right), F^{n}\left(x_{0}, y_{0}, z_{0}\right)\right)+\right. \\
d\left(F^{n+1}\left(y_{0}, z_{0}, x_{0}\right), F^{n}\left(y_{0}, z_{0}, x_{0}\right)\right)+d\left(F^{n+1}\left(z_{0}, x_{0}, y_{0}\right), F^{n}\left(z_{0}, x_{0}, y_{0}\right)\right] \leq \\
\leq k^{n+1} \cdot\left[d\left(F\left(x_{0}, y_{0}, z_{0}\right), x_{0}\right)+d\left(F\left(y_{0}, z_{0}, x_{0}\right), y_{0}\right)+d\left(F\left(z_{0}, x_{0}, y_{0}\right), z_{0}\right)\right] .
\end{gathered}
$$

This implies that $\left\{F^{n}\left(x_{0}, y_{0}, z_{0}\right\}_{n \in \mathbb{N}}\right.$ is a Cauchy sequence in $X$.

Similarly, following the same steps, we can prove that $\left\{F^{n}\left(y_{0}, z_{0}, x_{0}\right)\right\}_{n \in \mathbb{N}}$ and $\left\{F^{n}\left(z_{0}, x_{0}, y_{0}\right)\right\}_{n \in \mathbb{N}}$ are also Cauchy sequences in $X$.

Since $X$ is a complete metric space, there exist $\bar{x}, \bar{y}, \bar{z} \in X$ such that $F^{n}\left(x_{0}, y_{0}, z_{0}\right) \rightarrow$ $\bar{x}, F^{n}\left(y_{0}, x_{0}, z_{0}\right) \rightarrow \bar{y}, F^{n}\left(z_{0}, y_{0}, x_{0}\right) \rightarrow \bar{z}, \quad n \rightarrow \infty$.

Let $m>n$. Then:

$$
\begin{gathered}
d\left(F^{m}\left(x_{0}, y_{0}, z_{0}\right), F^{n}\left(x_{0}, y_{0}, z_{0}\right)\right) \leq \sum_{j=n}^{m-1} d\left(F^{j+1}\left(x_{0}, y_{0}, z_{0}\right), F^{j}\left(x_{0}, y_{0}, z_{0}\right)\right) \leq \\
\leq\left(k^{n-1}+k^{n}+\ldots+k^{m-n}\right) \\
=\frac{k^{n}-k^{m}}{1-k} \cdot\left[d\left(F\left(x_{0}, y_{0}, z_{0}\right), x_{0}\right)+d\left(F\left(y_{0}, z_{0}, x_{0}\right), y_{0}\right)+d\left(F\left(z_{0}, x_{0}, y_{0}\right), z_{0}\right)\right] \\
<\frac{k^{n}}{1-k} \cdot\left[d\left(F\left(x_{0}, y_{0}, z_{0}\right), x_{0}\right)+d\left(F\left(y_{0}, z_{0}, x_{0}\right), y_{0}\right)+d\left(F\left(z_{0}, x_{0}, y_{0}\right), z_{0}\right)\right]< \\
\left.\left.\left.x_{0}\right), y_{0}\right)+d\left(F\left(z_{0}, x_{0}, y_{0}\right), z_{0}\right)\right] .
\end{gathered}
$$

But $x_{n+1}=F\left(x_{n}, y_{n}, z_{n}\right), y_{n+1}=F\left(y_{n}, x_{n}, z_{n}\right)$ and $z_{n+1}=F\left(z_{n}, y_{n}, x_{n}\right)$, $F^{n}\left(x_{0}, y_{0}, z_{0}\right) \rightarrow \bar{x}, F^{n}\left(y_{0}, x_{0}, z_{0}\right) \rightarrow \bar{y}, F^{n}\left(z_{0}, y_{0}, x_{0}\right) \rightarrow \bar{z}, \quad n \rightarrow \infty$ and $F$ is orbitally continuous. Thus, applying maximum to the last relation, we get:

$$
\begin{gathered}
\max _{n \in \mathbb{N}}\left\{d\left(x_{n}, \bar{x}\right), d\left(y_{n}, \bar{y}\right), d\left(z_{n}, \bar{z}\right)\right\} \leq \frac{k^{n}}{1-k} \cdot\left[d\left(F\left(x_{0}, y_{0}, z_{0}\right), x_{0}\right)+\right. \\
\left.+d\left(F\left(y_{0}, x_{0}, z_{0}\right), y_{0}\right)+d\left(F\left(z_{0}, y_{0}, x_{0}\right), z_{0}\right)\right] .
\end{gathered}
$$




\section{EXAMPLES AND CONCLUSIONS}

Example 1. The Theorem 2 is more general than the fixed point theorem of Asgari and Mousavi [1] because of the new contractivity condition, fact illustrated by the following example:

Let $X=\mathbb{R}$, the metric $d(x, y)=|x-y|$, the relation $R$ on $X$ given by

$$
x R y \Leftrightarrow \frac{x-2 y}{3}=\frac{y-2 x}{3} .
$$

Let $F: X \times X \rightarrow X$ be defined by $F(x, y)=\frac{x-2 y}{3}$. So, $\forall(x, y) \in X^{2}$, F has the mixed $R$-monotone property, satisfies (2.1), but does not satisfy the contractivity condition in Asgari and Mousavi's fixed point theorem [1]. Indeed, assume that there exists $k \in[0,1)$ such that (1) holds. This means

$$
\left|\frac{x-2 y}{3}-\frac{z-2 t}{3}\right| \leq \frac{k}{2}[|x-z|+|y-t|], \forall(x, y) \in X_{R}(z, t)
$$

by which, for $x=z$, we get

$$
\frac{2}{3}|y-t| \leq \frac{k}{2}|y-t|
$$

which, for $y \neq t$, would imply $\frac{4}{3} \leq k$, where $k<1$, a contradiction. Now, we prove that (2.1) holds. We have

$$
\left|\frac{x-2 y}{3}-\frac{z-2 t}{3}\right| \leq \frac{1}{2}|x-z|+\frac{1}{3}|y-t|, \forall(x, y) \in X_{R}(z, t)
$$

and

$$
\left|\frac{y-2 x}{3}-\frac{t-2 z}{3}\right| \leq \frac{1}{2}|y-t|+\frac{1}{3}|x-z|, \forall(x, y) \in X_{R}(z, t) .
$$

By summing up these two relations, we get (2.1) with $k=\frac{5}{6}<1$.

Example 2. Let $X=\mathbb{R}$, the metric $d(x, y)=|x-y|$, the relation $R$ on $X$ given by

$$
(x, y, z) R(t, u, v) \Leftrightarrow x R t \wedge y R u \wedge z R v,
$$

where $x R t \Leftrightarrow x^{2}+2 x=t^{2}+2 t$ Let $F: X \times X \times X \rightarrow X$ be defined by $F(x, y, z)=$ $x^{2}-2 x-4$. So, $\forall(x, y, z) \in X^{3}$, we have :

$$
\begin{gathered}
X_{R}(x, y, z)=\{(x, y, z),(x, y+2, z),(x+2, y, z),(x+2, y+2, z),(x, y, z+2), \\
(x+2, y+2, z+2),(x+2, y, z+2),(x, y+2, z+2)\} . \\
F \times F\left(X_{R}(x, y, z)\right)=\{F \times F(x, y, z)\} \subseteq X_{R}(F \times F(x, y, z))
\end{gathered}
$$

So, $F$ has the mixed $R$-monotone property. Furthermore, $F$ is continuous and $\exists(-3,5,2) \in X \times X \times X$ such that $F(-3,5,2) \in X_{R}(-3,5,2)$. Therefore, the hypotheses of the Theorem 3 is satisfied. The tripled fixed points are: $(4,4,4),(4,-1,4)$, $(-1,4,4),(-1,-1,4),(4,4,-1),(-1,4,-1),(4,-1,-1),(-1,-1,-1)$. 
In their research, Asgari and Mousavi point and use the fact that the binary relation used in the previous results in the field doesn't have to be necessarily antisymmetric, reflexive or transitive. Thus, they use a reflexive binary relation, obtaining new, original results. In this paper, we took advantage of the concept of R-coupled fixed point and mixed R-monotone property of a mapping [1] to introduce new coupled and tripled fixed point results with respect to a reflexive relation, using symmetrical contractivity conditions, in the sense of Berinde et. al. [3], [5].

\section{ACKNOWLEDGEMENT}

The author wishes to thank the referees for the valuable suggestions leading to an improved version of the manuscript.

\section{REFERENCES}

[1] M. S. Asgari and B. Mousavi, "Coupled fixed point theorems with respect to binary relations in metric spaces," J. Nonlinear Sci. Applications, vol. 8, no. 2, pp. 153-162, 2015.

[2] H. Ben-El-Mechaiekh, "The Ran-Reurings fixed point theorem without partial order: a simple proof," J. Fixed Point Theory Appl., vol. 16, no. 1-2, pp. 373-383, 2014, doi: 10.1007/s11784015-0218-3.

[3] V. Berinde, "Generalized coupled fixed point theorems for mixed monotone mappings in partially ordered metric spaces," Nonlinear Anal., vol. 74, no. 18, pp. 7347-7355, 2011, doi: 10.1016/j.na.2011.07.053.

[4] V. Berinde and M. Borcut, "Tripled fixed point theorems for contractive type mappings in partially ordered metric spaces," Nonlinear Anal., vol. 74, no. 15, pp. 4889-4897, 2011, doi: 10.1016/j.na.2011.03.032.

[5] M. Borcut, M. Păcurar, and V. Berinde, "Tripled fixed point theorems for mixed monotone Kannan type contractive mappings," J. Appl. Math., pp. Art. ID 120203, 8, 2014, doi: $10.1155 / 2014 / 120203$.

[6] M. Edelstein, "An extension of Banach's contraction principle," Proc. Amer. Math. Soc., vol. 12, pp. 7-10, 1961, doi: 10.2307/2034113.

[7] T. Gnana Bhaskar and V. Lakshmikantham, "Fixed point theorems in partially ordered metric spaces and applications," Nonlinear Anal., vol. 65, no. 7, pp. 1379-1393, 2006, doi: 10.1016/j.na.2005.10.017.

[8] D. J. Guo and V. Lakshmikantham, "Coupled fixed points of nonlinear operators with applications,” Nonlinear Anal., vol. 11, no. 5, pp. 623-632, 1987, doi: 10.1016/0362-546X(87)90077-0.

[9] A. Petruşel and I. A. Rus, "Fixed point theorems in ordered l-spaces," Proc. Amer. Math. Soc., vol. 134, no. 2, pp. 411-418, 2006, doi: 10.1090/S0002-9939-05-07982-7.

[10] A. Ran and M. Reurings, "A fixed point theorem in partially ordered sets and some applications to matrix equations," Proc. Amer. Math. Soc., vol. 132, no. 5, pp. 1435-1443, 2004.

[11] B. Samet and M. Turinici, "Fixed point theorems on a metric space endowed with an arbitrary binary relation and applications," Commun. Math. Anal., vol. 13, no. 2, pp. 82-97, 2012. 
Author's address

Melánia-Iulia Dobrican

Technical University of Cluj-Napoca,North University Center at Baia Mare, Department of Mathematics, Victoriei Street nr. 76, 430122, Baia Mare, Romania

E-mail address: melania.cozma@yahoo.com 